\title{
A Materiomics Approach to Spider Silk: Protein Molecules to Webs
}

\author{
ANNA TARAKANOVA ${ }^{1}$ and MARKUS J. BUEHLER ${ }^{1,2}$ \\ 1.-Laboratory for Atomistic and Molecular Mechanics (LAMM), Department of Civil and Envi- \\ ronmental Engineering, Massachusetts Institute of Technology, 77 Massachusetts Avenue, \\ Cambridge, MA 02139, USA. 2.—e-mail: mbuehler@mit.edu
}

\begin{abstract}
The exceptional mechanical properties of hierarchical self-assembling silk biopolymers have been extensively studied experimentally and in computational investigations. A series of recent studies has been conducted to examine structure-function relationships across different length scales in silk, ranging from atomistic models of protein constituents to the spider web architecture. Silk is an exemplary natural material because its superior properties stem intrinsically from the synergistic cooperativity of hierarchically organized components, rather than from the superior properties of the building blocks themselves. It is composed of beta-sheet nanocrystals interspersed within less orderly amorphous domains, where the underlying molecular structure is dominated by weak hydrogen bonding. Protein chains are organized into fibrils, which pack together to form threads of a spider web. In this article we survey multiscale studies spanning length scales from angstroms to centimeters, from the amino acid sequence defining silk components to an atomistically derived spider web model, with the aim to bridge varying levels of hierarchy to elucidate the mechanisms by which structure at each composite level contributes to organization and material phenomena at subsequent levels. The work demonstrates that the web is a highly adapted system where both material and hierarchical structure across all length scales is critical for its functional properties.
\end{abstract}

\section{INTRODUCTION}

Proteins play a critical role as the building blocks of many complex hierarchical biological materials. $^{1-5}$ The diversified functionality of protein-based materials is often a result of hierarchical structure, rooted in the molecular composition and expanding the material design space to incorporate multiple levels. ${ }^{4,6,7}$ Often, the underlying composition of such systems is founded on weak building blocks, which are abundantly available, accessible to build largescale materials through low-energy processing, or have an innate capacity to self-assemble. The goal of the emerging field of materiomics ${ }^{8}$ is to characterize material systems on multiple length scales, for example by bridging weak building block components to particular mechanical behaviors on the macroscale. Spider silk is an excellent demonstration of a biological protein fiber where the hierarchical structure-exhibiting weak hydrogen bonding at its core-regulates material behavior on multiple length scales. The hierarchies in silk's material composition are illustrated in Fig. 1a. Silk is a terrific prototype of Nature's design, interesting particularly because of its exceptional qualities, even surpassing high energy-absorbing materials such as Kevlar and carbon fiber, and providing an extremely lightweight alternative. ${ }^{9-12}$ A combination of high tensile strength on par with steel (at $1 \mathrm{GPa}$ to $2 \mathrm{GPa}^{11}$ ) and extensibility (up to $60 \%$ maximum strain ${ }^{11}$ ) results in superior toughness and exceeding performance when normalized by its density. Spiders utilize these unique material properties to construct geometrically organized web structures which serve a variety of functions, most significantly the effective capture of prey as well as for the construction of egg sacks and cocoons. The most widely studied silk, and the focus of our discussion, is dragline silk, ${ }^{13,14}$ which functions to provide a stable framework in orb-webs. ${ }^{9}$ For a 
(a)

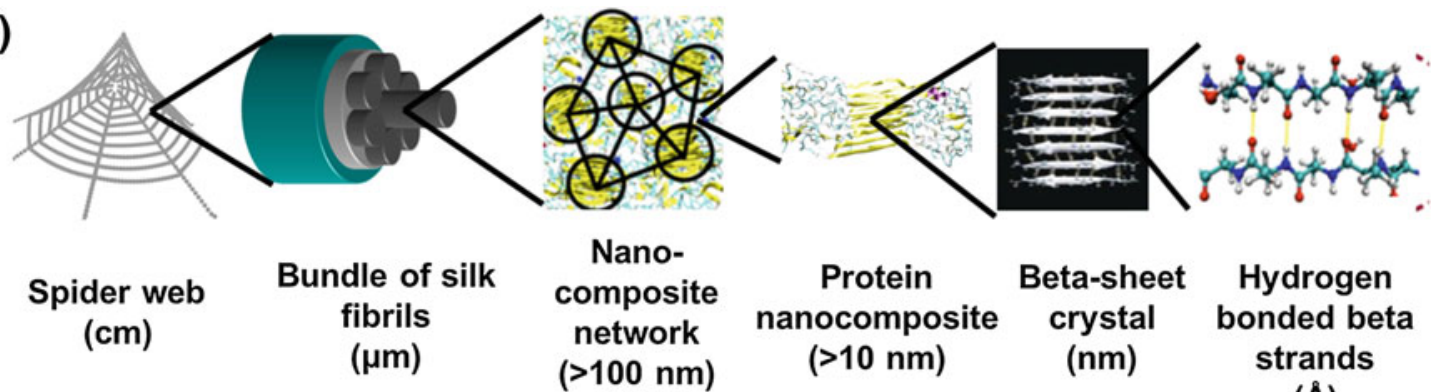

(A)

(b)

Silk structure identified using statistical methods

(E.g., Replica Exchange MD)

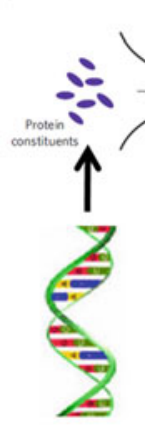

genetic information
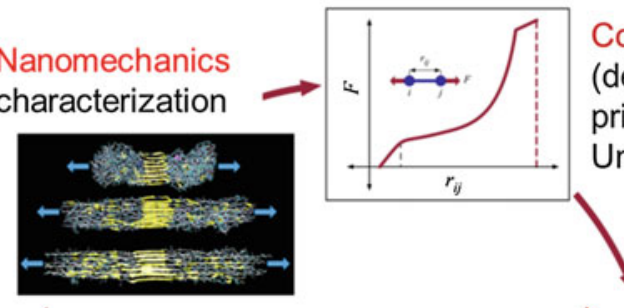

Constitutive behavior

(derived from molecular

principles, w/ mechanisms:

Unfolding, slip, failure)

Macroscale system

(web)

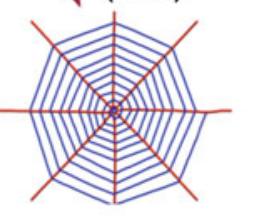

Web failure

(simulation, model and

in situ experiment)

Link between nano and macroscales
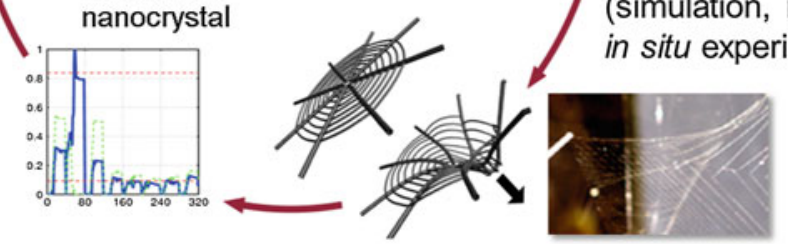

Fig. 1. Hierarchical structure of spider silk and multiscale approach used in our joint analysis using simulation and experiment. (a) Several hydrogen-bonded beta-strands make up a beta-sheet nanocrystal, where several beta-sheet nanocrystals are embedded in semi-amorphous domains consisting of more disordered subunits, together forming the protein nanocomposite structure. Protein chains are combined in a largerscale network that forms fibrils with diameters of around $100 \mathrm{~nm}$. Bundles of fibrils form the fibers with diameters of several micrometers, making up threads found in a spider web, the macroscopic structure. ${ }^{6,17}$ (b) A summary of the multiscale approach considered here for web analysis, to link the molecular to the macroscale. ${ }^{35}$ Silk protein structure, based on the information about its genetic makeup, is identified with methods such as replica exchange molecular dynamics and characterized to define a constitutive behavior. The molecularly derived behavior is incorporated into a macroscale web system. Web-scale deformation experiments, confirmed experimentally for validation, allow for analysis linking macroscale deformation to molecular mechanisms. The possibility of tracing varied molecular composition effects on macroscale behavior is realized experimentally through recombinant silk production mimicking the spider spinning process, outlining a powerful multiscale approach that incorporates experiment and simulation. ${ }^{39}$

complete description of such complex systems as spider orb-webs, a fundamental understanding of structure-function relationships across different length scales is required. Hence, spider orb-webs are an excellent model system for the methods of materiomics-involving multiscale simulation, theory, and experiment-to span all scales and arrive at a model that describes material function of the entire system as the interplay of the material's building blocks. ${ }^{15}$ Recent work has been conducted following such a bottom-up approach of material modeling, from atomistic models of silk protein to a macroscale orb-web system, and assessed along with experimental studies. In this article, we discuss a series of models to analyze the connection between composition and mechanical behavior across length scales, outlined in Table I. A notable result from these studies is that the observed exceptional properties of silk derive not from the superior material behavior at each level of hierarchy but rather from the combined or complementary function of building blocks at each length scale (Fig. 1b).

\section{RESULTS AND DISCUSSION}

Silk's lowest level of hierarchy is rooted in its primary protein structure, defined by a sequence of amino acid residues, which is responsible for subsequent folding mechanisms leading to the defining 


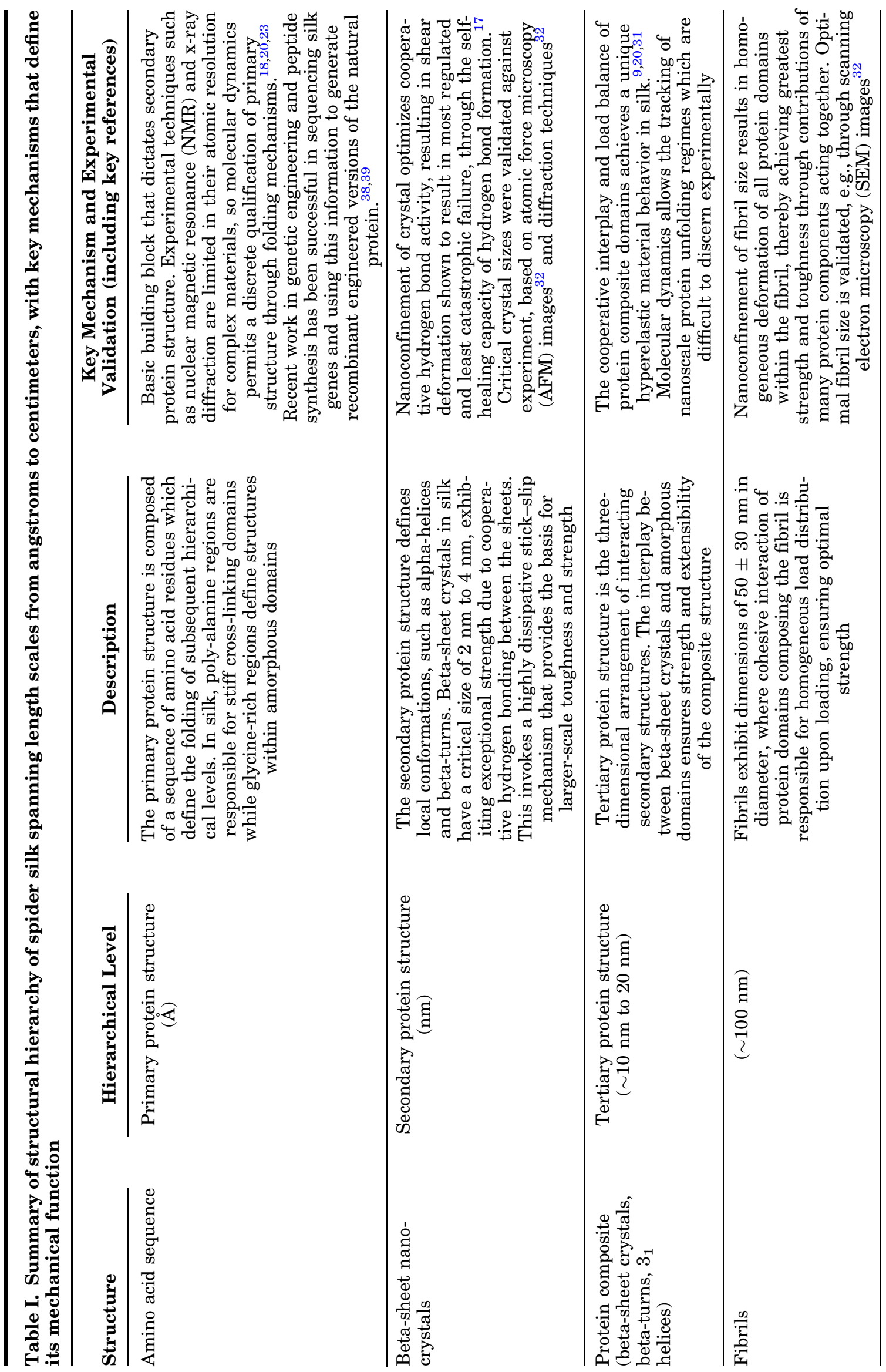


molecular structure. Residue segments form secondary structures, including crystalline beta-sheets, $3_{1}$ helices, and beta-turns. ${ }^{16}$ The chemical bonding within these structures determines the material properties of each, where dense hydrogen bonds compose the stiffer crystals, while dispersed hydrogen bonds join together extendable helices and betaturns. Three-dimensional arrangements of the secondary structures form a higher-order protein structure, where beta-sheet crystals are embedded in the amorphous meshwork of less orderly structures. Protein chains combine into silk fibrils that bundle together into the fibers forming threads in the web.

In what follows, each scale is considered through a three-stage analysis based on a series of hierarchical models. For each reviewed model we describe how it is developed through a bottom-up approach, linking more sophisticated lower length-scale parameters (the building blocks of the system for a subsequent hierarchical level) to coarser, larger length scales. Mechanical characterization is then considered as a means to assess functional properties of this material at each level. The third stage of the analysis links lower length-scale composition to higher length scales and appropriate deformation mechanisms. Essentially, stratification of different levels of hierarchy using this analysis develops a powerful feedback loop where the bottom-up modeling approach catalyzes the insights we gain at each layer of the material ladder (Fig. 1b). We conceive that the ability to analytically describe multiple levels of hierarchical behavior from building blocks opens the opportunities for creating tunable materials, with the possibility of controlling properties at multiple scales simultaneously. Such analysis projects the possibility of new design paradigms in areas of application ranging from highperformance fibers and multifunctional materials produced from artificially spun silk or derived materials, ${ }^{2,3}$ to novel material platforms for tissue engineering or even structural applications in the defense or aerospace industry. ${ }^{4,5}$

\section{Molecular Structure and Mechanics of Silk}

To study the link between chemical composition, structure, and mechanical behavior at the molecular scale, a model was created using a bottom-up molecular dynamics approach to identify native structures of the silk protein. ${ }^{17}$ The outline of this process is depicted in Fig. 2, where we start from primary structures of silk proteins with the amino acid sequence of the Nephila clavipes dragline MaSp1 protein (in one-letter amino-acid code) GG AGQGGYGGLGSQGAGRGGLGGQGAGAAAAAAG GAGQGGYGGLGSQGAGRGGLGGQGAG. ${ }^{18}$ The starting configuration in simulations of the composite protein structure consists of a lattice of an antiparallel arrangement in one direction and a parallel arrangement in the other, based on previous 
findings and mimicking the natural silk spinning process that involves elongational flow and alignment of protein chains due to mechanical force (Fig. 1b). ${ }^{3,17-20}$ Simulations were carried out with Langevin dynamics using CHARMM $^{21}$ and the EEF1.1 force field based on the Gaussian solvent exclusion model ${ }^{22}$ which leaves the potential for further equilibration in full-explicit solvent models once a reliable structure has been identified. ${ }^{20,23}$ Replica exchange molecular dynamics, ${ }^{24}$ an enhanced sampling method to explore a much longer time scale than accessible to conventional molecular dynamics, was employed here as a means to predict native structures, by overcoming energy barriers and local minima. ${ }^{25}$ Results were validated with experimental data by comparing dihedral angles of glycine and alanine residues through Ramachandran plots (Fig. 2c, d), showing that the computational predictions yield excellent results when compared with empirical analyses. ${ }^{26,27}$ Further details can be found in the original paper. ${ }^{20}$ Further analysis of the resulting structures showed that the poly-alanine region and adjacent poly-glycine-alanine segments formed a stiff orderly cross-linking

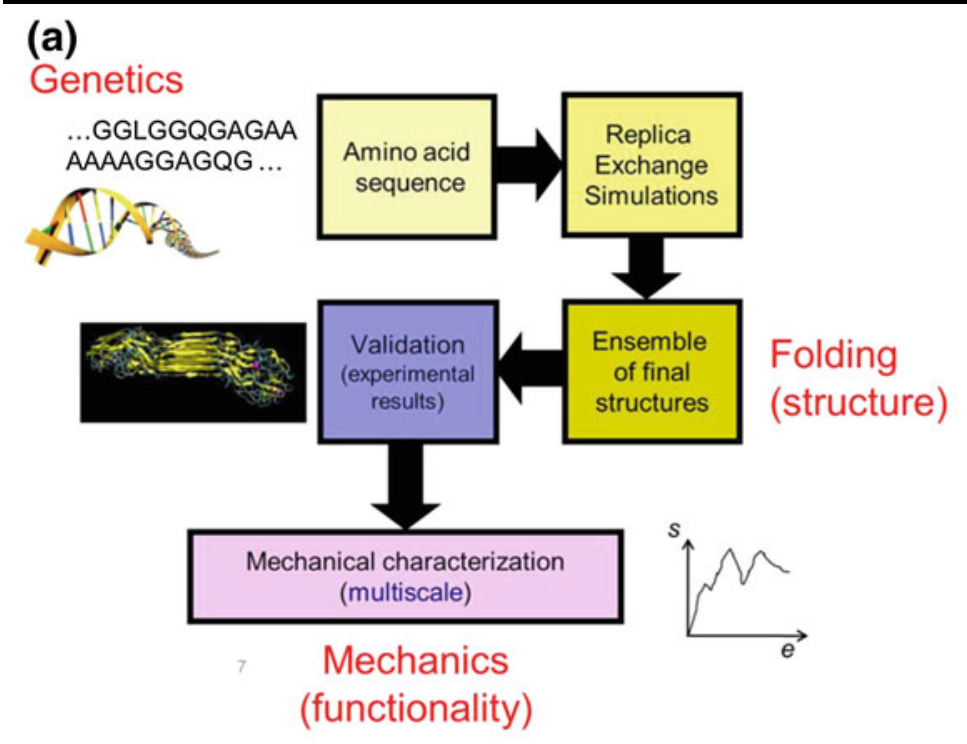

(b)
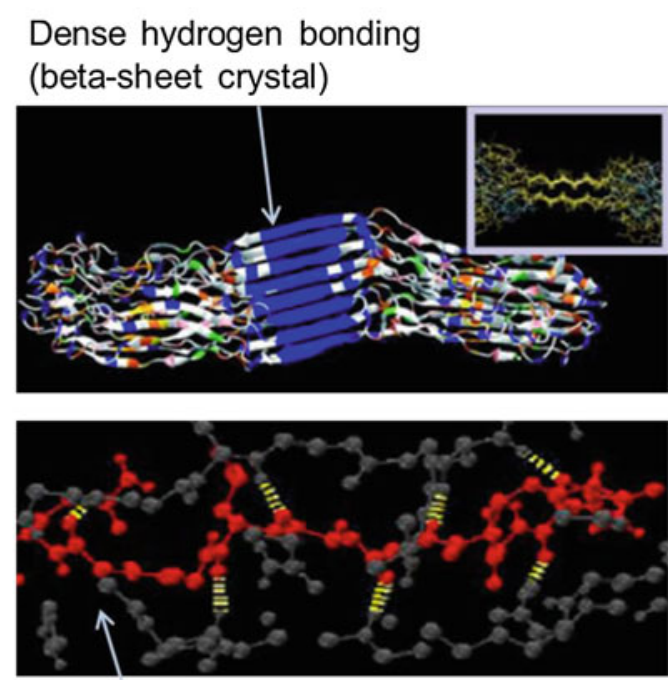

Irregular, dispersed hydrogen bonding (semi-amorphous)
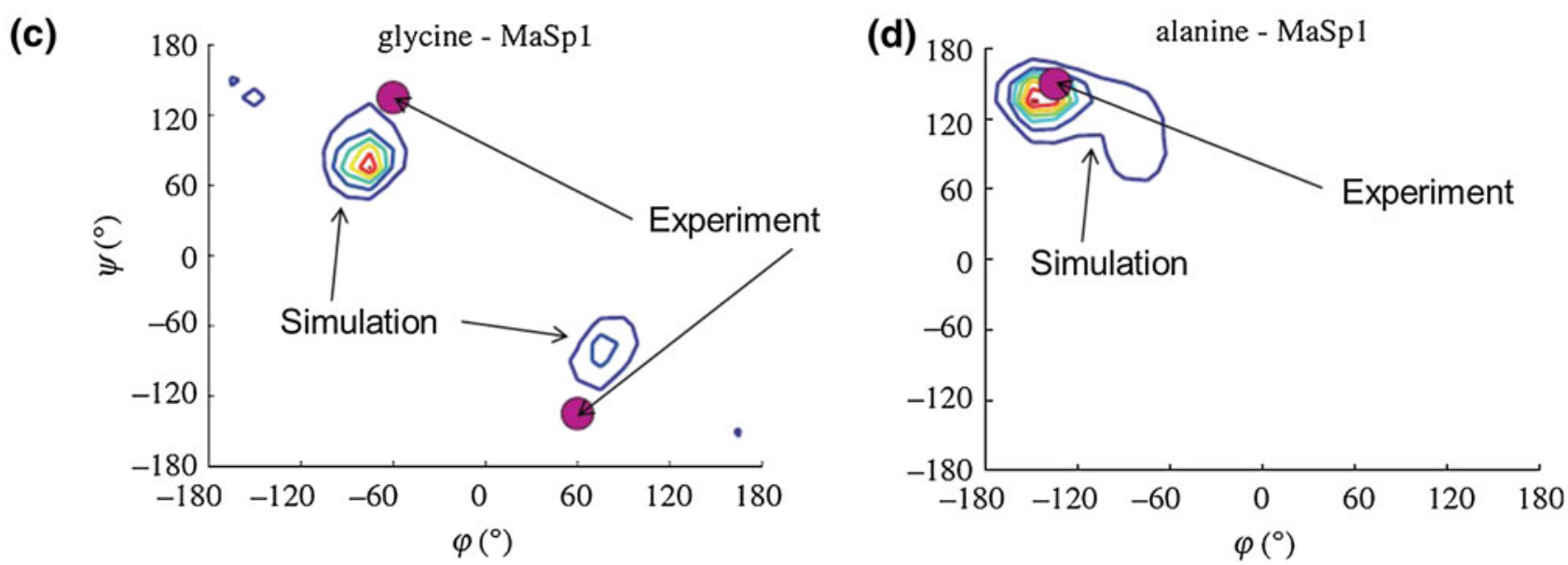

Fig. 2. Approach for protein structure identification and experimental validation. ${ }^{18,20,23}$ (a) The building blocks of silk proteins are a series of monomer units, consisting of a poly-alanine region between two glycine-rich domains (repeated up to 100 times in each chain). Replica exchange molecular dynamics simulations were conducted at different temperatures to determine the ensemble of most likely low-temperature native structures of small assemblies of repeat units, resembling a unit cell of silk. The resulting configurations were validated against experimental data of structure and composition. ${ }^{26}$ The resultant structures then allow for mechanical characterization on multiple scales as described in the subsequent figures. (b) Detailed view of the hydrogen bonding structure within different domains is identified with the model. Dense hydrogen bonding is found in stiff beta-sheet crystals and is responsible for their strength. Dispersed hydrogen bonding is found in amorphous regions, leading to a more irregular shape, and from a mechanical point of view, greater extensibility (as shown below). (c, d) As an example of structure validation, Ramachandran maps were used to compare the dihedral angles of glycine and alanine residues. Phi and psi refer to angles around $\mathrm{N}-\mathrm{C}_{\alpha}$ and $\mathrm{C}_{\alpha}-\mathrm{C}$ bonds. Shaded circles represent experimental values, while maps refer to distributions of angles derived from the model, both of which show angle values that match rather well. Figure modified from prior work. ${ }^{20,23}$ 
domain of the beta-sheet crystal, embedded in a semi-amorphous matrix formed by two surrounding GGX repeat regions. In fact, this model first identified the secondary structure composition of the amorphous regions, showing a lack of alpha-helix conformations, but rather a disorderly mixture of structures resembling $3_{1}$ helices and beta-turns, supporting a series of earlier experimental investigations. ${ }^{26,28-30}$

Silk's extraordinary properties on the macroscopic scale ultimately stem from the balance of strength and extensibility at the molecular scale. To assess the function of different protein domains, molecular-level deformation mechanisms of the protein composite were examined. ${ }^{20}$ These studies revealed that the balance is achieved through the combination of distinct secondary structure units in silk protein, where the interplay of the two constituent domains characterizes the nanoscale deformation mechanisms of the emerging nanocomposite structure. To qualify the cooperative function of the two main domains, representative structures were mechanically sheared at a constant loading rate, and resultant protein unfolding mechanisms were observed (Fig. 3) ${ }^{20}$ It was seen that, initially, the amorphous domain unfolds, as hydrogen bonds break and the beta-turn content decreases. Beta-turns within the amorphous domains provide hidden length, leading to the great extensibility and toughness observed in silk. A comparison between a series of native structures displayed an enhanced initial stiffening regime and an increase in extensibility for structures with a higher turn content. ${ }^{20}$ The source of this observation is an increased density of hydrogen bonds (leading to early stiffening) with increased turn

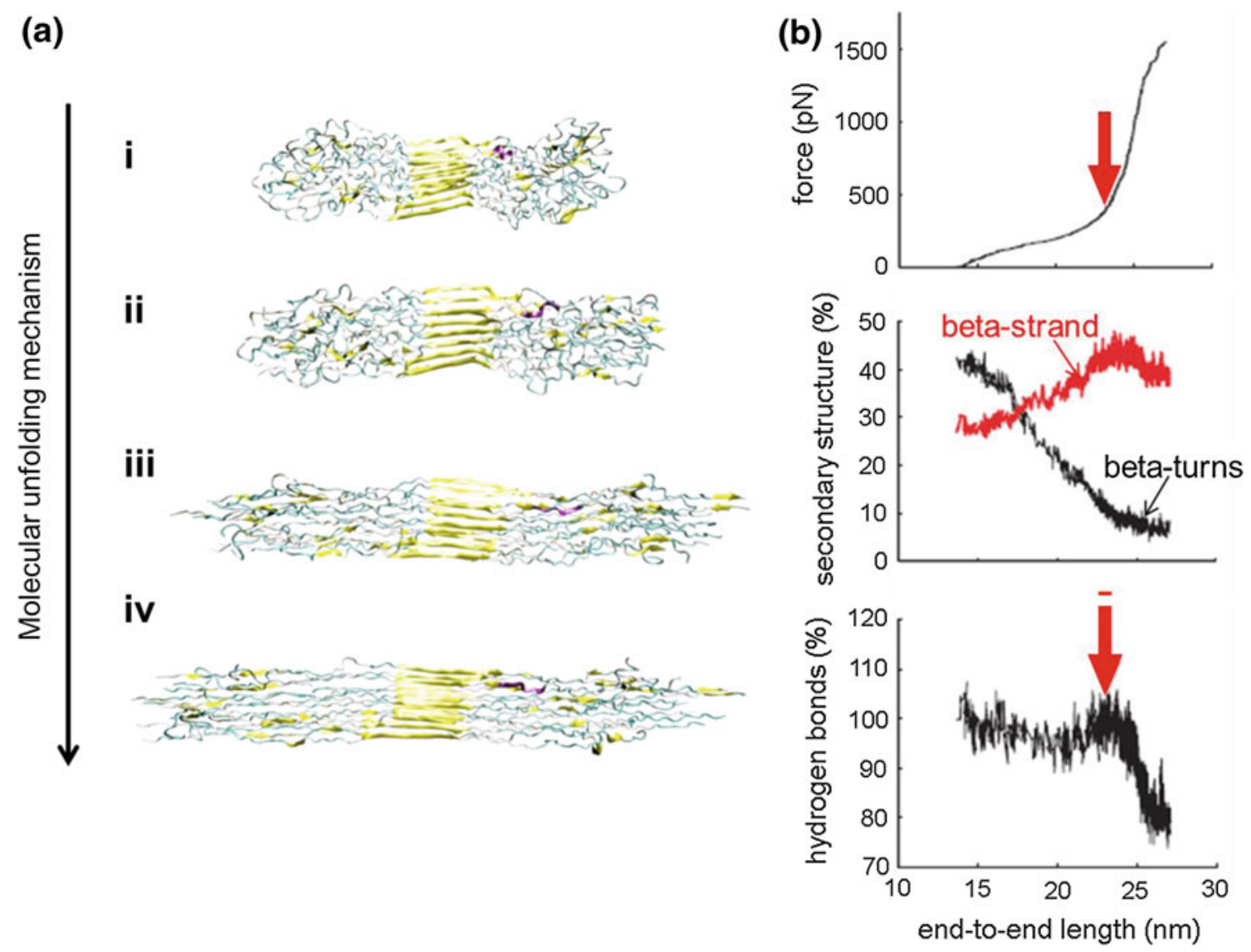

Fig. 3. Molecular unfolding mechanism of the protein nanocomposite and associated nanomechanical and structural properties as a function of increasing end-to-end length. ${ }^{20,31}$ (a) Qualification of the four-stage deformation mechanism of a single silk repeat unit. Initially (i), the protein nanocomposite consists of coiled semi-amorphous domains exhibiting hidden length embedding a stiff beta-sheet crystal. As the protein begins to unfold (ii), amorphous domains unravel, revealing their hidden length. As the amorphous domains stretch, new hydrogen bonds are created and beta-crystal units begin to form within the amorphous domains, sustaining the stress on the system as amorphous regions are extended to their limit (iii). A final regime is observed as covalent bond rupture forces many hydrogen bonds to break rapidly within the amorphous domains (iv), initiating sliding of beta-strands which ultimately leads to failure. (b) Quantification of unfolding regimes. The top figure shows the characteristic nonlinear force-displacement curve for stretching the protein nanocomposite, where the arrow indicates a final stiff regime as covalent bonds of the backbone rupture. The second figure indicates the percent secondary structure components within the protein composite upon deformation, where crystals (indicated by beta-strand content) form in the amorphous regions as beta-turns unwind. The final figure shows (arrow) the point at which hydrogen bonds within the amorphous domains begin to break dramatically, ultimately leading to failure of the system. A key result from these studies was that the beta-sheet crystal is the key factor in defining the mechanical strength of the composite, whereas the semi-amorphous phase controls the extensibility of the material. ${ }^{31}$ 
formation (increased extensibility). As the structure is further extended, beta-sheet crystals form in the amorphous domains, bearing the exerted stress. Stiffening occurs as covalent bonds extend and hydrogen bonds rupture in the amorphous regions. Failure comes in as hydrogen bonds break in the crystalline regions, which triggers the sliding of beta-sheet strands. This coherent interaction among different domains ${ }^{31}$ within the silk protein results in a nonlinear constituent behavior of silk comparable to experimental studies ${ }^{32}$ of silk threads, achieving high strengths from the stiff crystal components, and improved extensibility and toughness resultant from constituents of the amorphous region.

These findings motivated an examination of the crystal domain to key in on the underlying structure responsible for its superior stiffness. In fact, experiments suggested that nanocrystal size, moderated by the reeling speed of the silk, was the primary factor attributing their superior strength and toughness to the properties of cross-linking crystal domains. To understand the details of the underlying mechanisms, we surveyed the particular assembly and mechanical response of the nanocrystal. Hereby, beta-sheet crystals act as stiff orderly crosslinks whose underlying chemical structure is defined by assemblies of hydrogen bonds and, as established in a wide range of experimental and theoretical papers, presenting one of the weakest types of chemical bonds in Nature. These "inferior" building blocks together contribute to a structure that is essentially responsible for holding together the amorphous domains, allowing significant extension of the amorphous regions to proceed without rupture, reinforcing the polypeptide strands, and thus functioning as load-transferring units as seen in Fig. 3. To understand the governing structure responsible for crystal strength, beta-sheet nanocrystals were built by truncating the original system to four beta-strands and replicating to achieve different sizes. ${ }^{17}$

The performance of the different sized crystals was compared in pull-out simulations, where a middle strand was loaded with a constant pulling velocity. Material properties, including pull-out strength, toughness, and resilience of the system, were quantified, as seen in Fig. 4a. Smaller crystals exhibited superior properties up to a critical (optimal) size of $2 \mathrm{~nm}$ to $4 \mathrm{~nm}$, as a result of the difference in deformation mechanism on the molecular scale. In small crystals, a stick-slip motion was observed as hydrogen bonds broke and reformed. The self-healing ability of smaller crystals owing to the continuous reformation of hydrogen bonds protects the crystals from catastrophic failure as hydrogen bonds are shielded from exposure to water which facilitates rupture. In contrast to soft and brittle larger crystals which fail catastrophically by crack-flaw formation and consequent bending, smaller crystals exhibited shear deformation
(Fig. 4b). Snapshots in Fig. 4b show uniform breaking of hydrogen bonds connected to the middle stand of the smaller crystal governed by a stick-slip mechanism. The effective capacity of hydrogen bonds to resist failure by self-healing in conjunction with nanoconfinement at the crystal scale results in strong, stiff crystals of characteristic size, as verified experimentally. ${ }^{32}$ Thus, the cooperative assembly of weak hydrogen bonds shaping geometry at the nanoscale exemplifies a hierarchical bridging mechanism between the angstrom and the molecular scale.

\section{Silk Fibril Mechanics}

The characteristic behavior derived from silk protein studies is the key to understanding behavior and function at both the fibril and spider web scales. ${ }^{33}$ To examine this issue we invoked a coarsegrained model of the protein network combining semi-amorphous and crystalline domains, where the particle spacing corresponds to the distance between beta-sheet domains, at roughly $10 \mathrm{~nm} .{ }^{33} \mathrm{It}$ is important to note that the coarse-grain mesoscale model incorporates the material behavior at the protein scale derived from atomistic simulations into the bead-spring network, reaching length scales of micrometers without losing information about the underlying molecular mechanisms. To characterize the mechanical properties of individual fibrils with various types of defects and under different geometrical constraints, four loading scenarios were considered (Fig. 5a). A series of in silico tensile tests were conducted for varying fibril size $H$. Each fibril model contains a crack running through $50 \%$ of the respective length, to account for localized stress concentrations that occur as a result of defects in naturally occurring fibrils. Failure stress and strain were recorded for varying loading conditions and different fibril sizes, and compared with experimental data for three types of silk fibers. Results in Fig. 5b show that a critical length scale exists, defined as $H^{*}=50 \pm 30 \mathrm{~nm}$, at which the molecular properties of the protein network are most effectively scaled up to the micrometer scale in comparison with experimental stress-strain graphs typical of silk. Indeed, the length scale of $H^{*}=$ $50 \pm 30 \mathrm{~nm}$ is quite similar to experimentally measured fibril diameter lengths on the order of $20 \mathrm{~nm}$ to $150 \mathrm{~nm}$, as shown in Fig. 5c. ${ }^{14,32,34}$

The underlying mechanism responsible for this remarkable behavior is a homogeneity of stress distributions at fibril sizes approaching the critical length scale. ${ }^{33}$ Hence, as the fibril size approaches $H^{*}$, the majority of the material contributes to resisting failure, exhibiting failure stresses corresponding to the protein unfolding regimes III and IV depicted in Fig. 3. In the analysis of deformation fields at the crack tip in fibrils of different sizes, ${ }^{33}$ the authors found increased delocalization of stress at the crack tip as fibrils reached the critical fibril 

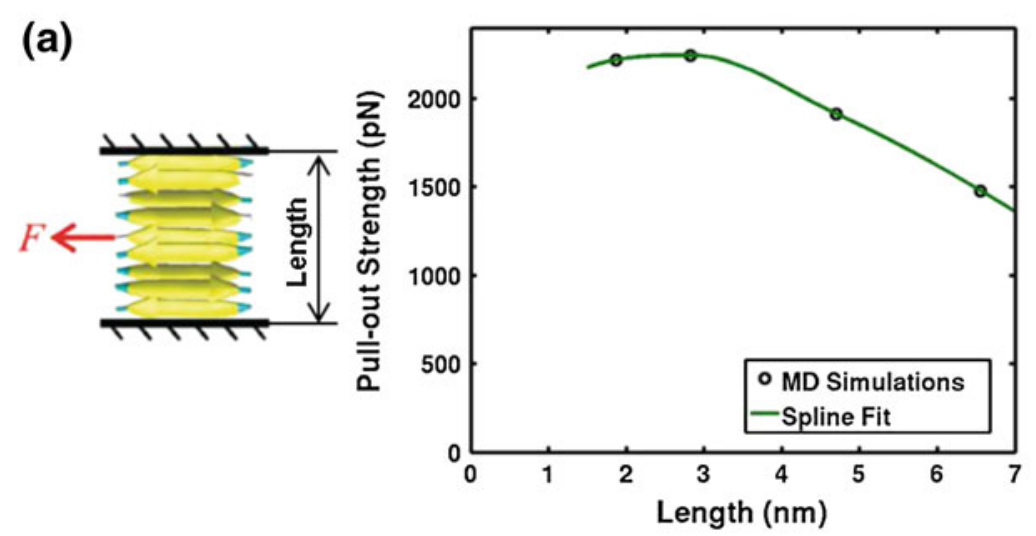

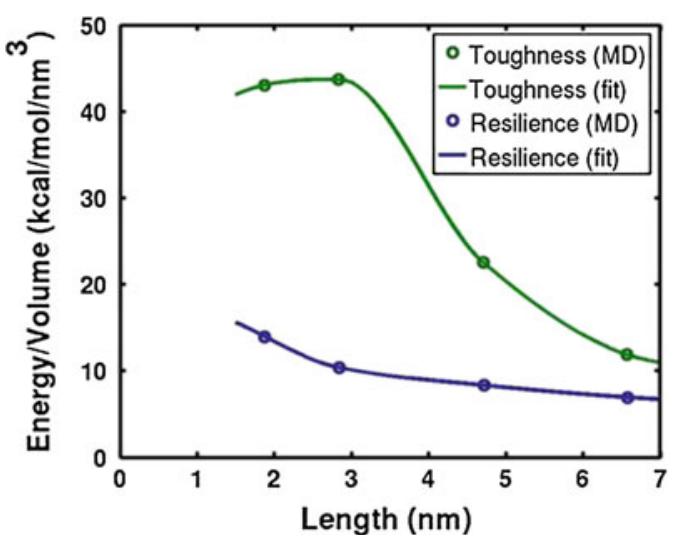

(b)

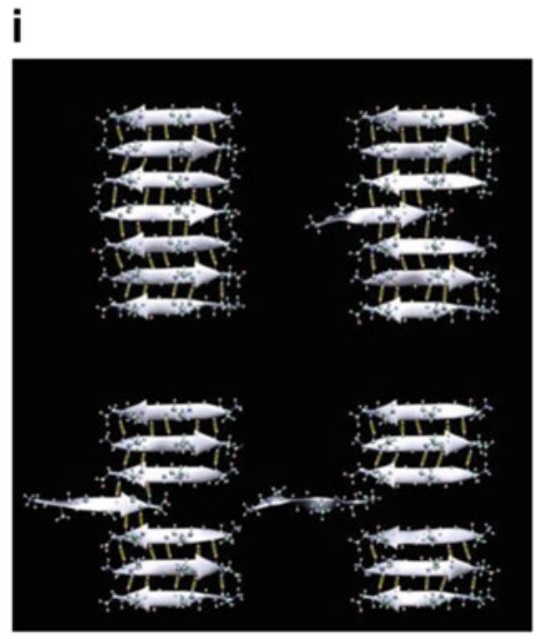

stick-slip deformation (robust) ii

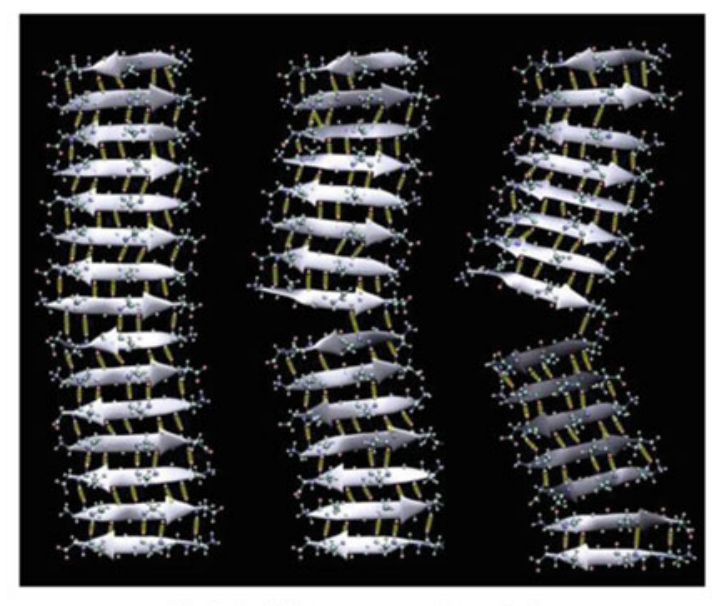

"brittle" fracture (fragile)

Fig. 4. Mechanical characterization of deformation of different sized beta-sheet crystals. (a) A beta-sheet is loaded horizontally at a middle strand. Toughness, resilience, and pull-out strength are shown as functions of varying beta-sheet crystal length (geometry indicated in the left part). ${ }^{17}$ An optimal size for enhanced mechanical properties is found between $2 \mathrm{~nm}$ and $4 \mathrm{~nm}$ crystal size. (b) Representative deformation mechanisms for small $(2.83 \mathrm{~nm})$ and large beta-sheet crystals $(6.56 \mathrm{~nm})$. Shear-dominated deformation is visible for small crystals, while larger crystals deform primarily through bending. Beta-strands in smaller-sized crystals experience homogeneous bond strain, leading to stick-slip deformation of hydrogen bonds. Thus, shear found in smaller crystals is more robust as a result of the self-healing capacity of hydrogen bonds to reform after rupture, as a consequence of their inherent weakness. This shows that the weak chemical building blocks are chiefly responsible for the load-withstanding capacity of smaller beta-crystals, and turn a weakness into strength by invoking highly dissipative deformation mechanisms. Figure modified from prior work. ${ }^{17}$

size $H^{*}$, resulting in completely homogeneous deformation where the entire sample reaches uniform stress concentrations before failure, pointing to a cohesive material mechanism to improve performance. The synergy of multiple protein components within the fibril takes advantage of the effective strength and extensibility of silk protein units, yielding superior toughness moduli of $190 \mathrm{MJ} / \mathrm{m}^{3}$ to $200 \mathrm{MJ} / \mathrm{m}^{3}$, 33 observed for fibrils of critical length scales, favorably comparable to the range of experimental findings of $100 \mathrm{MJ} / \mathrm{m}^{3}$ to $350 \mathrm{MJ} / \mathrm{m}^{3}$ across different species (Fig. $5 \mathrm{~d}$ ). ${ }^{10}$ Interestingly, a similar strength-enhancing nanoconfinement mechanism operates at the beta-sheet nanocrystal scale, but at that scale the mechanism is attributed to hydrogen bond cooperativity and rupture profile within the crystal, rather than the combined action of entire protein domains to resist load. However, the similarity of the basic concepts behind these mechanisms is apparent, and reflects the universality of structure-function relationships in hierarchical biomaterials more generally.

\section{Spider Web Mechanics}

The final, overarching hierarchical level we consider is a spider web model that describes silk fibers as a coarse-grained collection of beads assembled in a typical web geometry, ${ }^{35}$ where the material behavior is derived directly from atomistic simulation of the protein composite as reviewed above (Fig. 6). The derived mechanical response of the protein nanocomposite is linked to the deformation of a spider web upon simulated impact by prey, relating macroscale deformed web orientations to constituent protein domain states. We trace a direct 
(a)
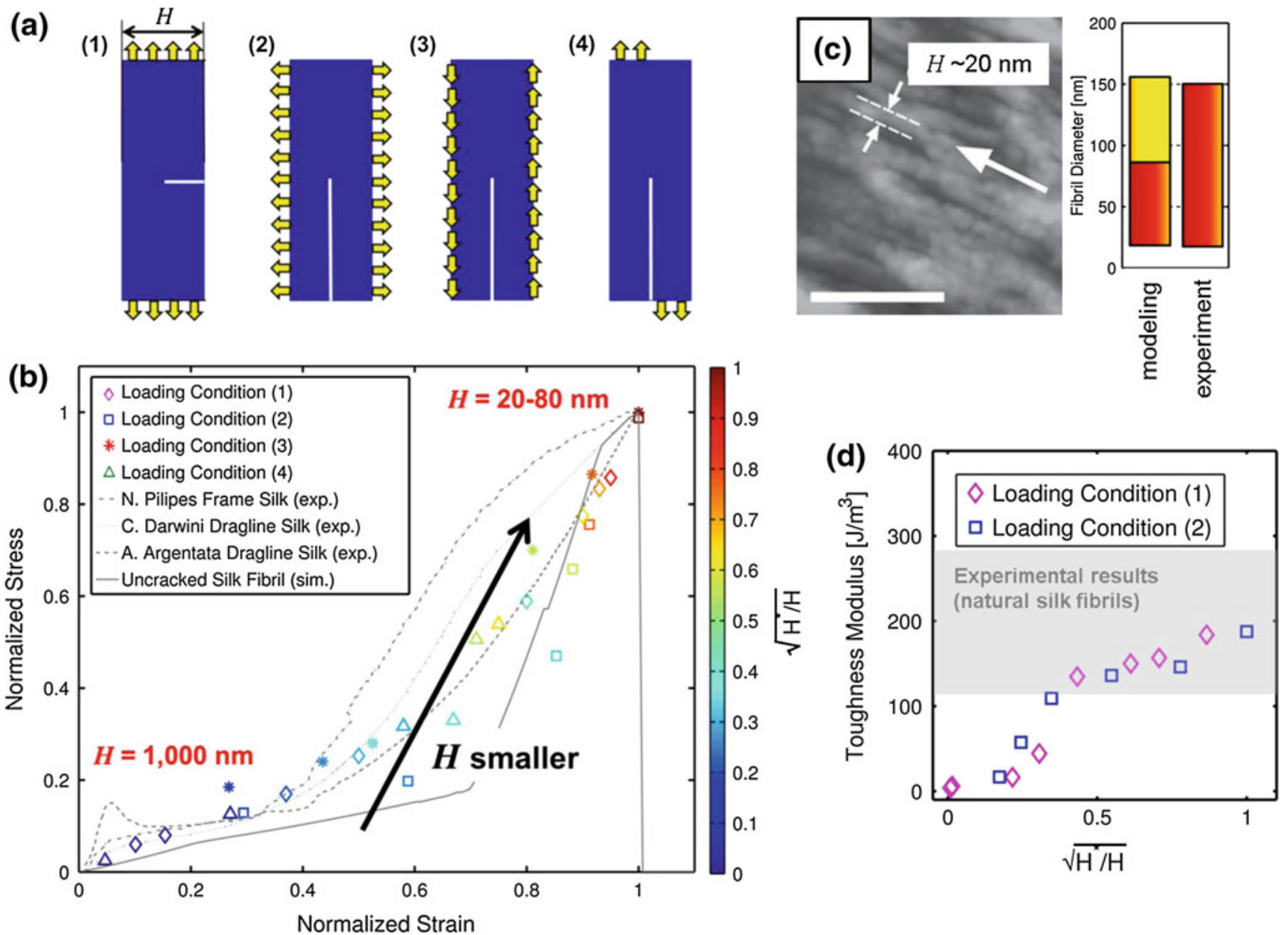

Fig. 5. Analysis of mechanical properties of silk fibrils and dependence of the failure strain and failure stress on the fibril diameter $H$, with comparison to experimental results. ${ }^{33}$ (a) Four loading conditions are considered for a two-dimensional network representative of silk protein fibrils, to include shear and tensile loading under varied fibril diameter $H$. A crack is introduced in each loading mode to represent naturally occurring flaws in the material. Fibril length $L$ is kept constant while the fibril width $H$ is varied. (b) Dependence of failure stress and strain on fibril size for the four loading conditions in panel (a). The gradient represents variation in fibril size where 0 is $H \gg H^{*}$ and 1 represents sizes approaching a characteristic size $H^{*}$, as size varies from $\sim 1000 \mathrm{~nm}$ to $\sim 50 \mathrm{~nm}$. Experimental data of three different silks are included for comparison. Solid line corresponds to loading profile of an uncracked fibril. Optimized material behavior is reached as fibril size approaches $H^{*} \approx 50 \pm 30 \mathrm{~nm}$. (c) Experimental image of a silk fibril (for Bombyx mori silk), ${ }^{34}$ indicating a diameter of $20 \mathrm{~nm}$ (scale bar $150 \mathrm{~nm}$ ). Reprinted with permission from Du et al., ${ }^{34}$ copyright (C) 2011 by John Wiley and Sons. (d) Modulus of toughness considered for varying fibril sizes under loading conditions (1) and (2). Both approach natural silk fibril values of $100 \mathrm{MJ} / \mathrm{m}^{3}$ to $350 \mathrm{MJ} / \mathrm{m}^{3}$ (indicated in shaded block in the graph) as the fibril size approaches the critical size $H^{*}$. Figure modified from prior work. ${ }^{33}$

connection established between the molecular unfolding mechanisms and the effective capacity of the web to resist load and mitigate damage through localization of failure. We note that this model does not account for the intermediate fibril scale intentionally, to gain a fundamental-level understanding of deformation. We justify this choice by noting that atomistically derived material behavior compares well with empirical studies of silk threads. ${ }^{32}$ The web is modeled by an arithmetic spiral, ${ }^{35}$ with radial threads extending from the center for support. The architecture and geometry are borrowed from the natural orb-web design, ${ }^{36}$ as illustrated in Fig. 6a. Threads consist of particles connected by springs, with equilibrium spacing of $1 \mathrm{~cm}$, exhibiting behavior derived from mechanical characterization of the protein composite (Fig. 6b). This modeling implementation allows for a direct relation between molecular configuration and web deformation state. Both radial and spiral threads are modeled based on the dragline silk behavior, though in Nature these silks exhibit different material properties. We note that, optimally, spiral threads would be modeled separately, but as no atomistic models exist this is done as an approximation. This issue has been addressed in previous work ${ }^{35}$ where approximated spiral thread behavior, based on dragline silk, was compared with an empirically derived material model, where both models exhibited a similar response upon loading.

Here we focus on one case study that was performed with the above-described web model. For mechanical characterization of the web, a spring load at constant velocity of $0.007 \mathrm{~m} / \mathrm{s}$ is applied to a 
(a)
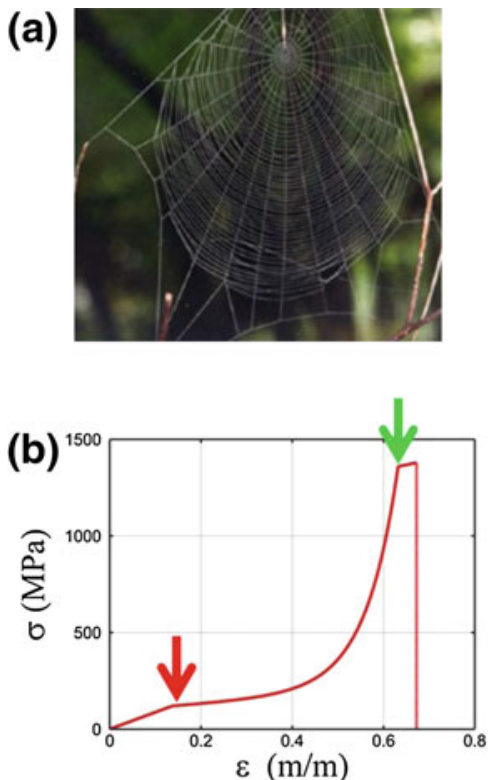

$\varepsilon(\mathrm{m} / \mathrm{m})$

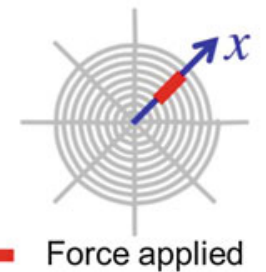

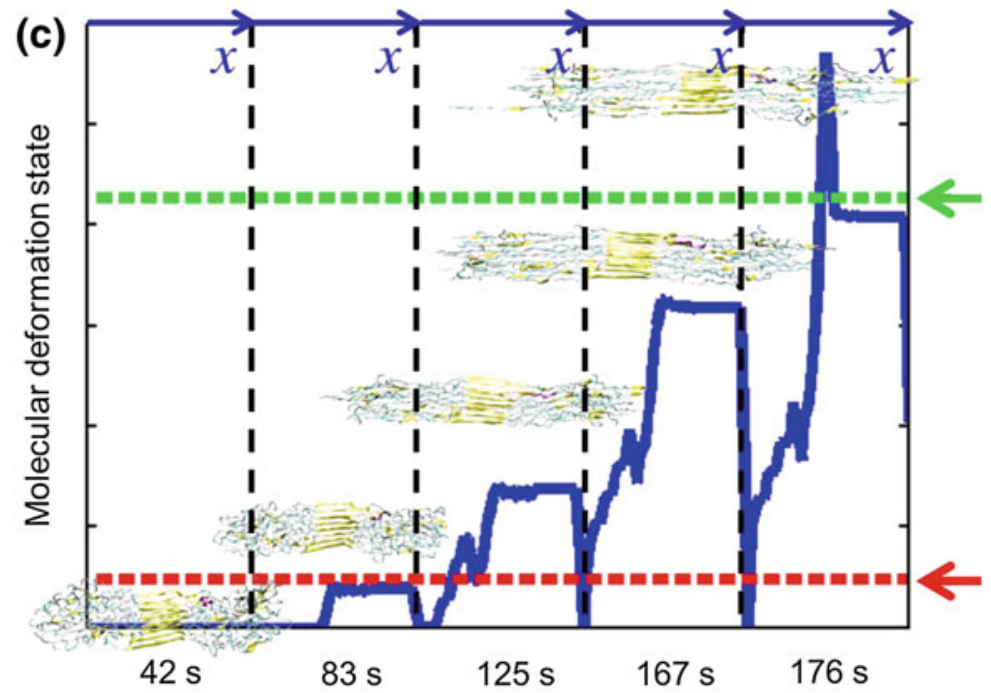

Time as web deforms
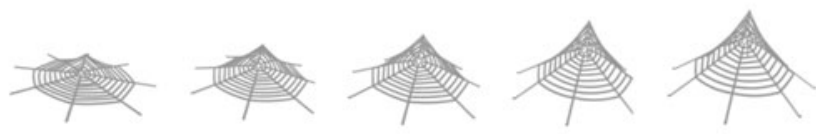

Fig. 6. Mechanical behavior of dragline spider silk, and web response upon localized loading of radial thread. (a) Geometry of typical spider orbweb, from Cyclosa simplicicauda. Image of the web reprinted with permission from Swanson et al., ${ }^{36}$ copyright (C) 2001 by John Wiley and Sons. (b) Threads in the web are modeled as particles connected via springs, exhibiting a stress-strain behavior parameterized from atomistic simulation of silk protein and as shown in the stress-strain plot. ${ }^{20,35}$ The arrow on the left corresponds to the yield point, where amorphous domains begin to unfold as hydrogen bonds break. The arrow on the right demonstrates the point at which beta-sheet nanocrystals begin to exhibit stick-slip deformation (molecular mechanisms identified as shown in Fig. 3). The deformation field in the thread is plotted in panel (c), also marking the thread where the local force is applied. (c) Deformation fields in a radial thread, under web loading with constant velocity of $0.002 \mathrm{~m} /$ $\mathrm{s}$. The figure shows the molecular deformation state along the radial thread, depicted for five time steps (separated by dashed lines). The molecular deformation states correspond to the five snapshots of the web shown in the lower part of the plot (web deformation tracked from the onset of deformation to just prior to failure). The bottom and top dashed lines correspond to the deformation states at the yield point and onset of the stick-slip behavior of beta-sheet crystals, respectively. The snapshots of protein constituents correspond to the molecular conformations present within the loaded radial at each time step. The first time step corresponds to onset of stretching where the protein has not begun to unfold. At $83 \mathrm{~s}$, the loaded amorphous regions within the protein domains of the loaded thread begin to stretch. At $125 \mathrm{~s}$, continued unfolding is observed until crystals begin to form in the amorphous region at $167 \mathrm{~s}$. At $176 \mathrm{~s}$, just prior to failure, a very small region in the vicinity of where loading is applied displays a peak in the deformation state, corresponding to the regime of slipping beta-sheet crystals.

single radial thread, mimicking the impact of prey striking the web or a piece of debris, until failure occurs, defined by the initial rupture of the loaded thread. The deformation proceeds for approximately 3 min (in real time) until the web breaks. The gradual application of load allows us to track various molecular-level deformation regimes. The development of molecular deformation states is shown in Fig. 6c, which also shows five representative snapshots during web deformation. The first configuration corresponds to the initial application of load where little deformation of the web is visible $(42 \mathrm{~s})$. The deformation state of the entire radial thread is at zero, corresponding to compact protein constituent domains (as illustrated by the corresponding snapshot), where no unfolding has yet occurred. As the load is applied, the web stretches to its second configuration (83 s). The composing protein structure approaches the yield point when amorphous domains begin to unfold. The following web configuration (125 s) corresponds to further unfolding of amorphous domains and the onset of rupture of hydrogen bonds within the domains. The fourth snapshot at $167 \mathrm{~s}$ describes a molecular phase where crystals begin to form and sustain the stress within the amorphous domains as chains are stretched to the limit. The final snapshot (176 s) is taken just before failure occurs. In parts of the loaded radial thread, beta-sheet nanocrystals begin to slip (indicated by the deformation states that reflect the surpassing of the top dashed line, marking the onset of stick-slip deformation). These molecular deformation mechanisms at the web scale are in fact responsible for the ability of the web to sustain load and resist catastrophic failure through a sacrificial mechanism. ${ }^{35}$ In comparison with other common engineering material behaviors, for example, linear-elastic and elastic-perfectly-plastic, the 
characteristic softening at the yield point followed by stiffening at large strain is responsible for localization of damage in a loaded web. This has significant evolutionary implications, ${ }^{11,37}$ ensuring that debris or prey too large for the web to support will not cause irreparable damage, costing the spider energy to rebuild. Furthermore, the regulation of damage through control of material behavior has implications beyond webs to any structural design which would benefit from contained damage. Looking forward, the entirety of a bottom-up design paradigm illustrated here, where function can be manipulated through alteration of structure on different hierarchical scales, presents opportunities in design of enhanced and novel synthetic, biomimetic and biological materials that provide a wide array of material function at low cost.

\section{CONCLUSIONS}

We have outlined a series of models describing increasing levels of hierarchy within a spider web, and illustrated how such multiscale modeling complements experimental efforts in developing a thorough understanding of the mechanics of a spider web. The strength of each model lies in its catalytic nature. In chemistry, a catalyst functions to speed up reaction rates, bridging the reactant and product states in solution. In hierarchical modeling of materials, the model allows for a convenient link between length scales, bridging scales through pinpointing assembly and deformation mechanisms. At the molecular level, the model takes as input the sequence of residues, yielding structure, size characterization, material behavior parameters, and mechanisms as output. The amino acid sequence dictates the chemistry of higher-order secondary protein structures of silk: domains of low and high density of hydrogen bonds are achieved in simulation. Low-density hydrogen bonding is present in the coiled, elastic domains, while denser hydrogen bonding results in the mechanism of nanoconfinement, which directs the characteristic size and strength of crystals composing silk. The two domains establish a collaborative interaction contributing complementary function: strength and extensibility. The unique strength of the bottom-up analytic approach is, in addition to a more complete and accurate system description, the potential to link higher-order scales to fundamental components at lowest hierarchical levels. The material behavior of silk is played out on two scales: the fibril and the web. At the fibril scale, nanoconfinement that stems from size-dependent homogeneity in load distribution dictates the fibril size. We make the distinction between nanoconfinement at this scale, which depends on the synergism of protein constituents, and the nanoconfinement of beta-crystals, a result of cooperative hydrogen bonding. At the web scale, we trace the ability of the web to effectively resist load to molecular mechanisms.
A combined assessment of computational and experimental studies, using silk as an example, provides an understanding of the challenges currently faced within complex material analysis. Experimental methods lack the power to accurately, or definitively, describe the molecular composition of silk at atomistic levels, as a result of imaging technique limitations. Computational methods, though accessible at atomistic scales, require experimental validation on the macroscopic scale. We have outlined a series of models complementing and describing multiscale phenomena from a material hierarchy perspective. We propose that, through analyzing bridging hierarchies within complex materials, material scientists will be able to narrow the knowledge gap existing in the description of materials on computational and experimental sides. We envision that such a scale-bridging approach to studying hierarchical materials, including materials other than silk such as natural and synthetic fibers, will facilitate better understanding at all levels of material composition and provide tools for effectively manipulating these properties to achieve superior material qualities for application in a variety of fields, from structural engineering to medicine. As the understanding gap narrows, we seek direct methods to be able to implement manipulation of material upon all length scales.

\section{ACKNOWLEDGEMENTS}

Primary support from ONR, AFOSR, and NSF is acknowledged. A.T. acknowledges support from a Robert A. Brown Presidential Fellowship.

\section{REFERENCES}

1. P. Fratzl and F.G. Barth, Nature 462, 442 (2009).

2. F.G. Omenetto and D.L. Kaplan, Science 329, 528 (2010).

3. S. Rammensee, U. Slotta, T. Scheibel, and A.R. Bausch, Proc. Natl. Acad. Sci. 105, 6590 (2008).

4. M.J. Buehler and Y.C. Yung, Nat. Mater. 8, 175 (2009).

5. C. Vepari and D.L. Kaplan, Prog. Polym. Sci. 32, 991 (2007).

6. M.J. Buehler, Nano Today 5, 379 (2010).

7. D. Sen and M.J. Buehler, Sci. Rep. 1, 35 (2011).

8. M.J. Buehler and S. Cranford, Nanotechnol. Sci. Appl. 3, 127 (2010).

9. J.M. Gosline, P.A. Guerette, C.S. Ortlepp, and K.N. Savage, J. Exp. Biol. 202, 3295 (1999).

10. I. Agnarsson, M. Kunter, and T.A. Blackledge, PLoS ONE 5, e11234 (2010).

11. B.O. Swanson, S.P. Anderson, C. Digiovine, R.N. Ross, and J.P. Dorsey, Integr. Comp. Biol. 49, 21 (2009).

12. F. Vollrath, Nature 466, 319 (2010).

13. F. Vollrath, Int. J. Biol. Macromol. 24, 81 (1999).

14. F. Vollrath, T. Holtet, H.C. Thogersen, and S. Frische, Proc. R. Soc. B Biol. Sci. 263, 147 (1996).

15. D. Spivak, T. Giesa, L. Wood, and M.J. Buehler, PLoS ONE 6, e23911 (2011).

16. M. Heim, L. Romer, and T. Scheibel, Chem. Soc. Rev. 39, 156 (2010).

17. S. Keten, Z. Xu, B. Ihle, and M.J. Buehler, Nat. Mater. 9, 359 (2010).

18. S. Keten and M.J. Buehler, Appl. Phys. Lett. 96, 153701 (2010).

19. B. Ma and R. Nussinov, Protein Sci. 11, 2335 (2002). 
20. S. Keten and M.J. Buehler, J. R. Soc. Interface 7, 1709 (2010).

21. B. Brooks, R. Bruccoleri, B. Olafson, D. States, S. Swaminathan, and M. Karplus, J. Comput. Chem. 4, 187 (1983).

22. T. Lazardis and M. Karplus, Genetics 35, 133 (1999).

23. G. Bratzel and M.J. Buehler, J. Mech. Behav. Biomed. Mater. (2011). doi:10.1016/j.jmbbm.2011.07.012.

24. Y. Sugita and Y. Okamoto, Chem. Phys. Lett. 314, 141 (1999).

25. M. Feig, J. Karanicolas, and C.L. Brooks, J. Mol. Graph. Model. 22, 377 (2004).

26. J.D. van Beek, S. Hess, F. Vollrath, and B.H. Meier, Proc. Natl. Acad. Sci. U S A 99, 10266 (2002).

27. J. Kummerlen, J.D. van Beek, F. Vollrath, and B.H. Meier, Macromolecules 29, 2920 (1996).

28. L.W. Jelinski, Curr. Opin. Solid State Mater. Sci. 3, 237 (1998).

29. G.P. Holland, M.S. Creager, J.E. Jenkins, R.V. Lewis, and J.L. Yarger, J. Am. Chem. Soc. 130, 9871 (2008).
30. J.E. Jenkins, M.S. Creager, R.V. Lewis, G.P. Holland, and J.L. Yarger, Biomacromolecules 11, 192 (2010).

31. A. Nova, S. Keten, N.M. Pugno, A. Redaelli, and M.J. Buehler, Nano Lett. 10, 2626 (2010).

32. N. Du, X.Y. Liu, J. Narayanan, L. Li, M.L. Lim, D. Li, Biophys. J. 91, 4528 (2006).

33. T. Giesa, M. Arslan, N.M. Pugno, and M.J. Buehler, Nano Lett. 11, 5038 (2011).

34. N. Du, Z. Yang, X.Y. Liu, Y. Li, and H.Y. Xu, Adv. Funct. Mater. 21, 772 (2011).

35. S.W. Cranford, A. Tarakanova, N. Pugno, and M.J. Buehler, Nature 482, 72 (2012).

36. B.O. Swanson, T.A. Blackledge, and C.Y. Hayashi, J. Exp. Zool. A Ecol. Genet. Physiol. 307A, 654 (2007).

37. F. Vollrath and P. Selden, Annu. Rev. Ecol. Evol. Syst. 38, 819 (2007).

38. N.A. Ayoub, J.E. Garb, R.M. Tinghitella, M.A. Collin, and C.Y. Hayashi, PLoS ONE 2, e514 (2007).

39. A. Rising, M. Widhe, J. Johansson, and M. Hedhammar, Cell. Mol. Life Sci. 68, 169 (2010). 